\title{
Modelling wrinkling interactions produced by patterned defects in metal thin films
}

\author{
E.A. Flores-Johnson ${ }^{1}$, Timothy J. Rupert ${ }^{2}$, Kevin J. Hemker ${ }^{3}$, Daniel S. Gianola ${ }^{4}$, Yixiang Gan ${ }^{1, *}$ \\ ${ }^{1}$ School of Civil Engineering, The University of Sydney, Sydney, NSW 2006, Australia \\ ${ }^{2}$ Department of Mechanical and Aerospace Engineering, University of California, Irvine, CA 92697-3975, United States \\ ${ }^{3}$ Department of Mechanical Engineering, Johns Hopkins University, Baltimore, MD 21218, United States \\ ${ }^{4}$ Department of Materials Science and Engineering, University of Pennsylvania, PA 19104-6391, United States
}

\begin{abstract}
Wrinkling patterns in freestanding metal thin films under tensile loading are investigated through finite-element simulations with experimental validation. Numerical simulations of the tensile testing of a thin film specimen with different arrays of holes were conducted. Good agreement between experiments and simulations was found for not only the spatial wrinkling pattern distributions, but also the pattern evolution over the different loading stages. The numerical results show that plasticity plays an important role in the evolution of wrinkling patterns and their associated interactions in $\mathrm{Al}$ thin films. It was found that the spacing between defects and defect size control the level of interaction between wrinkle branches generated by the defects. Strong interactions resulted in the merging of different wrinkle branches into one single wrinkle with a large displacement amplitude, a well-defined profile in the out-of-plane direction. The simulations proved to be robust in their descriptions of the experimentally observed wrinkling phenomenon and could be used to predict wrinkling in other hole-patterned thin film configurations. The interaction and merging of wrinkle branches from multiple holes indicate that the resulting wrinkle patterns can be manipulated by using different defect configurations to achieve a desired wrinkled "microstructure".
\end{abstract}

Keywords: Freestanding metal thin film; Wrinkling patterns; Patterned defects; Post-buckling analysis; Finite-element simulations.

*Corresponding author: Tel.: +61 29351 3721; fax: +61 29351 3343. E-mail address: yixiang.gan@ @ydney.edu.au

\section{Introduction}

Wrinkling patterns are commonly encountered in thin film structures and membranes subjected to local compressive stresses, either in loaded freestanding conditions $[1,2]$ or via mechanical mismatches with substrates [3-7]. The pattern and its evolution can be controlled through different means of stress generation, e.g., applied lateral displacements [8, 9], temperature variation [10], surface tension via liquid-solid interaction [11], and controllable residual stresses [12-15]. Specific wrinkling patterns can be designed to achieve desired functions in thin film systems, via changing surface morphology and roughness [16-18], wetting and adhesive properties [11, 18], tuneable mechanical properties [19], and generating micro-fluidic channels [20]. These wrinkling patterns manifest in structural [14, 21], biological [6, 22], electronic (e.g., 2D materials) [23], and metrology applications [3, 9], but most modelling efforts treat materials as defect-free. Defects in such 2D and layered materials are common and may control the nucleation and propagation of wrinkling patterns [24-28]. However, despite intense research efforts on the mechanical response of thin films ranging from necking instabilities to wrinkling mechanisms [29-33], all of which are crucial for thin film heterostructures and flexible electronics applications, the influence of defects within thin membranes in controlling the wrinkling patterns has not received much attention, particularly in metallic thin films.

To address this gap in current knowledge, we perform numerical simulations to model freestanding metal thin films with different types of pre-patterned defects subjected to remote uniaxial stretches with an emphasis on the development and evolution of the resulting wrinkling patterns and their interactions. We modelled intentionally-defected metallic thin films with arrays of holes to investigate 
how the spatial and configurational variation of the defects affects the overall wrinkling patterns and interactions of individual wrinkle branches. These numerical simulations are validated by comparing with experiments on patterned submicron Al thin films, showing that the simulations faithfully reproduced the wrinkling patterns. Our simulations use the finite element method (FEM) and have been performed with consideration of the full elastic-plastic behaviour of the material and initial residual stresses, to not only predict the evolution of complex wrinkling patterns and their interactions but also to access the stress and strain fields.

\section{Methodology}

Here, we consider Al thin films as typical examples of metallic films, which are commonly used in many technological applications such as in stretchable electronics [34]. Wrinkling patterns in thin metallic films have not been studied as widely as polymer membranes, with the important difference between these two classes of thin films being the occurrence of plasticity. In this letter, we will perform a full elastic-plastic numerical analysis for various geometries of patterned defects to investigate the wrinkling patterns and their interactions, with detailed experimental validation. Due to the difficulty of experiments on these films, the tensile tests were performed only on three different arrays of holes. We will focus on the additional information provided by numerical simulations and analyse the results systematically, such as the inhomogeneous stress and strain fields and the role of defect spacing, which are of critical importance for the understanding of interactions between wrinkles.

\subsection{Finite-element modelling}

To investigate the effect of defects on wrinkling in tensile testing of $\mathrm{Al}$ thin films, we constructed FEM models using the software Abaqus [35] with an implicit solver. The geometry and dimensions of the thin film model are shown in Fig. 1. The models were meshed using conventional quadrilateral shell elements (S4R) with a large-strain formulation, allowing transverse shear deformation. Although the minimum size of the shell elements is approximately 15 times larger than the film thickness, it is smaller than the typical expected wavelength of the wrinkling pattern as observed in experiments. The mesh was refined in regions near the holes, where larger changes in stresses are expected (Fig. 1(a)). A mesh sensitivity analysis showed that this level of mesh refinement was deemed sufficient for our numerical model.

To represent the pre-stressed state of these thin films before the tensile test owing to residual stresses that develop during film deposition, we applied an eigenstrain via a virtual increase of film temperature resulting in an estimated equi-biaxial compression stress on the film. This approach has been successfully used in thin film buckling analyses [15, 36]. The magnitude of this equi-biaxial stress was estimated in the range between 10 to $50 \mathrm{MPa}$, which is similar in magnitude to reported values for stresses from the thermal expansion mismatch between $\mathrm{Al}$ and Si [37]. During the strainrelease process of this biaxial compressive stress, wrinkling can initiate in the simulations. Subsequently, the edges of the film were uniaxially stretched to approximately $30 \mu \mathrm{m}$.

For all simulations, we employed an elastic-plastic material model, which was based on experimental stress-strain curves from tensile testing of an $\mathrm{Al}$ specimen without patterned holes in the gage section [38]. It has been reported that the temporal evolution of grain size and distribution play important roles in governing the plasticity in this type of material [38]; however, we limited the scope of this work to a time-independent stress-strain curve for the material model, which was found to be sufficiently accurate for our numerical simulations. For Al films, the material model used a von Mises yield criterion with isotropic hardening. The following material properties were employed: Young's modulus $E=70 \mathrm{GPa}$, Poisson's ratio $v=0.34$, and yield strength $\sigma_{Y}=80 \mathrm{MPa}$ [38].

We performed instability calculations in our FEM model to understand the complex deformation state that arises in the thin film geometry. Thin stressed membranes are known to develop instabilities that cause the material to wrinkle out-of-plane. Buckling analyses were performed based on the Riks method $[39,40]$ due to the nonlinearity in geometry and plastic behaviour during the tensile loading. The Riks method uses the load magnitude as an additional independent parameter and it solves 
simultaneously for loads and displacements. Therefore, another quantity called arc length must be used to measure the progress of the solution [35]. The arc length is used along the static equilibrium path in the load-displacement space. This approach provides solutions in problems with unstable behaviour such as post-buckling analysis with snap-through response. In our simulations, an appropriate maximum arc length was chosen by trial and error to achieve convergence of the problem (typically using $0.1 \% \sim 0.2 \%$ of the total loading time).

\subsection{Experimental validation}

In addition to our FEM simulations, we performed experiments with different pre-patterned geometries to compare with our numerical results. Three types of patterned freestanding thin film specimens were fabricated for micro-tensile testing, using standard Si-based microfabrication techniques as described in detail in [41]. Photolithography was utilized in conjunction with a number of etching techniques ( $\mathrm{KOH}$ wet etching, deep reactive ion etching, and pulsed $\mathrm{XeF} 2$ ) to define features on a (100) silicon wafer. Backside wafer processing was used to produce silicon frames $(1 \mathrm{~cm}$ $\times 1 \mathrm{~cm}$ ), which provided a base for film deposition and served as sample grips for the handling and deformation of the films. The geometry and dimensions of the experimental testing frame are shown in Fig. 1(b). Frontside micromachining was used to define the tensile geometries with defined microholes shown in Figs. 1(c)-(e), referring to horizontal 2-hole $(\mathrm{H} 2 \mathrm{H})$, angled 2-hole $(\mathrm{A} 2 \mathrm{H})$, and 4-hole (4H) geometries, respectively. The patterned holes are all $100 \mu \mathrm{m}$ in diameter. The gage widths and lengths of the specimens were $700 \mu \mathrm{m}$ and $4 \mathrm{~mm}$, respectively. All films were deposited to thicknesses of 150 to $220 \mathrm{~nm}$ by using pulsed electron beam evaporation of $99.999 \%$ pure Al.

The tensile testing setup consisted of one fixed grip connected to a load train and another grip which could be moved either by a piezoelectric screw-driven linear picomotor (for tensile elongation) or a 5-axis motorized stage (for sample alignment). The 5-axis stage was used in unison with a stereoscopic microscope to precisely align the grips to each other. Next, the silicon sample frame was placed on top of the grips and aligned using the edge of the silicon die as a guide. A combination of UV-curing glue and Loctite 414 was used to adhere the specimen to the grips. The Si support strips were then cut using a Dremel rotary tool with a diamond-impregnated blade attached to a 3-axis stage. The rotary blade was carefully brought into contact with the strip and slowly advanced through the strip to prevent large vibrations or rotation of the die from damaging the thin film. The freestanding films were then pulled in tension using the picomotor. A nominal strain rate of $5 \times 10^{-5} \mathrm{~s}^{-1}$ was used for all tensile tests performed for this study. During loading, the deformed films were observed through a light microscope at different stages. The wrinkling patterns were all observed with a normal incidence light source. We will later compare these experimental results with our numerical simulations.

\section{Results and discussion}

Here, we first present numerical results with different material properties to show the effect of plasticity on the development of wrinkling patterns followed by an explanation of how to interpret the observed wrinkling patterns in both experiments and simulations depending on lighting conditions. Next, we compare experimental and numerical results and describe wrinkling patterns at different loading stages. Then, we focus on wrinkling interactions by presenting one-hole geometry and twohole geometries with progressively decreasing separations. Finally, we further study wrinkling interactions for different spatial arrangements of holes and defect size, to gain insight into more complex defect arrangements or patterns.

\subsection{Influence of plasticity and lighting}

Figures 2(a)-(c) compare wrinkling patterns for the $\mathrm{H} 2 \mathrm{H}$ array with different material properties. For the elastic-plastic material described in Section 2.1, well-defined wrinkling patterns (see out-ofplane deformation in the left subfigure in Fig. 2(a)) including wrinkling interactions in the film midsection are observed (Fig. 2(a)), which is in agreement with experimentally observed wrinkling patterns (Fig. 3(a)). However, for an elastic-perfectly plastic material (Fig 2(b)), the film is very "soft" and at very small nominal strains $(\sim 0.15 \%)$, shear bands originated at the defects, where plastic strain 
is larger than $0.2 \%$, are developed, which suggests that film failure will occur. For this reason, there is no opportunity for the wrinkle patterns to develop in an elastic-perfectly plastic material. When a purely elastic material is employed, the film is very "hard" and wrinkling patterns develop only at very large strains (>1.85\%, shown in Fig. 2(c)). Moreover, the wrinkling pattern amplitude is around five times smaller than that of the elastic-plastic case. These results demonstrate that plasticity plays an important role in the development of wrinkling patterns in the metal thin film studied here.

Now, we will use Figure 2(d)-(e) to explain the connection between the observed wrinkling patterns and lighting conditions, an important consideration when comparing our modelling predictions with the experimental results. If we focus on the area denoted by the red square in Fig. 2(a), we could intuitively say that the wrinkle pattern has 5 peaks (bright zones) and 6 valleys (dark zones) (Fig. 2(d)); however, if the lighting conditions change from normal incident light (Fig. 2(d)) to angled incident light (Fig. 2(e)), 3 peaks and 3 valleys are now seen. The blue and yellow curves in Figs. 2(d) and 2(e) are the actual and apparent out-of-plane displacement of the film, respectively, at the cross section of the wrinkle area. It can be seen that for the normal incident light case, the apparent wavelength of the wrinkle is double the length of the actual wavelength. For the angled incident light case, both actual and apparent wavelengths are the same; however, they are shifted 1/4 of a wavelength from each other. It is noted, however, that neither lighting conditions will give the real out-of plane profile that can only be obtained through simulations by observing to the $z y$ plane. This shows the advantages of taking a numerical approach to studying wrinkling since measuring the outof-plane displacement experimentally would be very difficult in such films. Thus, for the remainder of the paper, we will use the elastic-plastic model for Al material and focus on the normal incident light condition, corresponding to the actual material and testing conditions in the experiments.

\subsection{Deformation patterns of $\mathrm{H} 2 \mathrm{H}, \mathrm{A} 2 \mathrm{H}$ and $4 \mathrm{H}$ arrays}

Having isolated the effects of plasticity and experimental lighting conditions on the actual and perceived wrinkling patterns, respectively, we now analyse the role of defects in controlling the details of wrinkling. In Figure 3, the simulated wrinkling patterns (left columns) are compared at three different loading stages with the corresponding experimental observations (right columns) for the three pre-patterned film geometries, namely $\mathrm{H} 2 \mathrm{H}, \mathrm{A} 2 \mathrm{H}$ and $4 \mathrm{H}$ cases, respectively. For all cases, qualitative agreement between experiments and simulations can be found for not only the spatial distributions of wrinkling patterns but also the pattern evolution at different loading stages. It is noted that at large strains, the predicted extension of the wrinkles' branches is shorter than that observed experimentally, particularly for the A2H case. This could be explained by more localised plastic zones in the simulation, which results in shorter wrinkles' branches when compared to the experiment. In Fig. 3, we observe that parallel wrinkles in the direction of the tensile loading initiate at the holes and the wrinkle domains grow with increasing strain. Interestingly, in all cases, the holes are spanned by parallel wrinkles aligned with the loading direction, suggesting strong interactions of wrinkles that initiated at different holes. Stretched membranes are characterized by three distinct regions: taut or tense, wrinkled and slack or inactive [2]. These regions have been predicted for elastic stretched membranes with one centred hole or no holes $[2,42]$. The capability of our simulations to predict these regions for a film that undergoes large plastic deformation with multiple holes is demonstrated with the insets at the bottom of Fig. 3 for $0.31 \%$ strain. We used the principal stress criterion [43] to define these regions: taut $\left(\sigma_{2}>0\right)$, wrinkled $\left(\sigma_{2} \leq 0\right.$ and $\left.\sigma_{1}>0\right)$, and slack $\left(\sigma_{2} \leq 0\right.$ and $\left.\sigma_{1} \leq 0\right)$.

To show how wrinkling affects the stress field around the arrays of holes, Figure 4 shows contour plots of the in-plane minimum principal stress $\sigma_{2}$ for each hole-patterned configuration at four different applied strains. The equivalent plastic strain, $\varepsilon_{e q}$, is also shown at $0.31 \%$ strain. A strain of $0.0 \%$ means that only the pre-stressed condition is applied to the film. The stress at $0.0 \%$ overall strain can give us a good idea of where the film is likely to wrinkle based on each hole-patterned configuration. We can see that the areas with pre-existing low compressive stress (green colour) initiate wrinkling patterns during subsequent tensile loading. The second strain value in each case (second row) represents the onset of wrinkling. The third strain value in each case (third row) represents the strain at which the out-of-plane deformation profile does not significantly change with further loading. At this strain, strong interactions between wrinkle branches have occurred. However, 
we note that these strains occur in the unstable loading-displacement response region in the simulations employing the Riks method, thus precluding quantitative comparison between these strains for each case. Nevertheless, we may still use these simulations to illustrate the wrinkling pattern evolution. At $0.31 \%$ strain, we can see that $\varepsilon_{e q}$ accumulates and localizes above and underneath the holes. Those zones with extensive plastic deformation would presumably be precursors to crack initiation sites that would subsequently propagate normal to the direction of loading and leading to eventual failure. The remainder of this paper will focus on strains below this localization event to study the evolution and interaction of wrinkling patterns.

\subsection{Understanding wrinkling interactions: defect spacing}

The existence of defects in close proximity to each other can lead to complex interactions in the wrinkling patterns owing to nonlinearities arising from material plasticity and geometric effects. To serve as a benchmark for comparison, we first examine the simplest configuration by isolating a single hole. We term this the 1-hole case $(1 \mathrm{H})$, which is presented in Fig. 5 (left-most column) together with contour plots of $\sigma_{2}$ shown at four different strains. We can see that two branches are generated at each side of the hole at the onset of wrinkling, which extend diagonally away from the hole, following the path with pre-existing compressive stress (green colour) observed at $0.0 \%$ strain. With further tensile loading, the wrinkling branches extend further horizontally in the loading direction and two initially separated wrinkle branches on each side of the hole merge into a single large wrinkle. This is in contrast with the wrinkling of an elastic rubber sheet with one hole [42], as well as the wrinkles on the right side of the right hole in Fig. 2(c) (elastic case) in which wrinkle branches do not merge, suggesting that plasticity facilitates a longer-range interaction for wrinkles. This idea is supported by the elastic-perfectly case (Fig. 2(b)) in which the extension of the wrinkle branches is larger than that of the elastic-plastic case (Fig. 2(a)), which includes the effect of material hardening.

Figure 5(e) shows the out-of-plane displacement $z$ at transverse planes for different holes configurations. For the $1 \mathrm{H}$ case, the transverse plane is located at 1.5 diameters in length from the centre of the hole. It can be seen that the wrinkle amplitude increases with increasing strain. The change in the wavelength of the wrinkles with the increase of the strain is negligible. This suggests that plasticity may play an important role in the increasing amplitude within a specific branch by facilitating the merging of individual parallel wrinkles in the branch at the expense of increasing wavelength when compared to the elastic case (compare out-of-plane profiles in Figs 2(a) and (c)). This is in contrast with the observed behaviour of elastic rubber sheets, in which the amplitude of the wrinkles decreases after reaching peak values and the wavelength decreases monotonically with the increase of strain [44]. It can also be seen that the wrinkle pattern has a well-defined symmetry configuration (see blue dotted horizontal centreline).

We next systematically study the role of wrinkling interactions by simulating two-hole geometries with progressively decreasing separations. For the case where two holes are separated by three times the original distance (hole separation in the $\mathrm{H} 2 \mathrm{H}$ case), the wrinkling pattern for each individual hole at low strain appears similar to that of the $1 \mathrm{H}$ case. The stress field at the midsection of the specimen suggests that no interaction is happening between wrinkling branches; however, the branches extending towards the centre of the film are slightly larger than the branches extending towards the sides the film. This is confirmed by the larger region with high stresses (green colour) in these branches when compared to the branches extending towards the sides. This indicates that weak wrinkling interactions are developing between the holes. This is further supported by examination of the out-of-plane displacement profiles shown in Fig. 5(e). For the transverse plane near the left hole, we can see that the symmetry of the pattern has been affected. If we use the horizontal centreline near the centre of the hole as a reference (blue dotted), the wrinkle seems to have an anti-symmetry configuration; however, if we use the shifted horizontal centreline (purple dotted) as a reference, the wrinkle seems to have a symmetry configuration. This indicates that a transition to a strongly interacting regime for wrinkling may be happening near this particular defect spacing. The $z$ profile at the transverse midplane shows that the film has wrinkled in that area and the anti-symmetry mode of the profile suggests that weak wrinkling interactions have also occurred; however, the magnitude of $z$ 
is less than ten times smaller than that at the transverse plane near the hole. For the case of two holes separated by twice the original distance, wrinkling interactions are clearly happening and the wrinkling profiles at both transverse plane near the hole and midplane have an anti-symmetry configuration. For the case where the holes are most closely spaced $(\mathrm{H} 2 \mathrm{H})$, strong interactions between wrinkles are observed and a well-defined profile with anti-symmetry configuration is observed. Notably, the magnitude of $z$ for the $\mathrm{H} 2 \mathrm{H}$ case is similar to that of the $1 \mathrm{H}$ case. This suggests that a strong wrinkling interaction of four branches (two from each hole) behave as a single large wrinkle, which results in more strain release and plastic deformation in that location. This is in contrast with weaker interactions (cases with two holes spaced by twice and three times the original distance) in which strain release happens at four different branches weakly interacting. We can also see from the 2-hole cases that the presence of strongly interacting branches makes the wrinkling patterns near the hole more spatially uniform or "smoothed".

\subsection{Understanding complex wrinkling interactions: arrangements of holes and defect size}

We now extend the analysis to study the influence of the spatial arrangement of holes and defect size on the ensuing wrinkling patterns. The insets at the top of Fig. 6 show wrinkling patterns at $0.31 \%$ strain. Figures $6(\mathrm{a}-\mathrm{c})$ show the predicted out-of-plane displacement $z$ at the transverse midplane for the $\mathrm{H} 2 \mathrm{H}, \mathrm{A} 2 \mathrm{H}$ and $4 \mathrm{H}$ cases, respectively. For the $\mathrm{H} 2 \mathrm{H}$ and $4 \mathrm{H}$ cases, the wrinkle pattern has a well-defined anti-symmetry configuration (relative to the axial midplane denoted by the dotted blue line). Furthermore, the wrinkling pattern is more uniform for the $\mathrm{H} 2 \mathrm{H}$ case when compared to the $4 \mathrm{H}$ case. For the $\mathrm{A} 2 \mathrm{H}$, the wrinkling mode is not that obvious, with the subtleties of wrinkling profile evolving with increasing applied strain. While a symmetric mode can be observed if one considers a shifted centreline (vertical purple dotted line), an anti-symmetric one emerges relative to the original centreline (blue dotted line). This suggests that a stable state exists between symmetric and anti-symmetric modes. These types of configurations have been observed for silicon rubber sheets [42] and classified as "in-between" states, which are in equilibrium. Although the wrinkles are also aligned with the loading direction in the A2H case (See Fig. 3), this asymmetry results in very irregular wrinkling patterns (Fig. 6(b)). It appears that the anti-symmetry evident in the $4 \mathrm{H}$ case is also determined by the strong interactions of the wrinkle branches as observed for the 2-hole cases in Fig. 5. However, the number of peaks and valleys in the $4 \mathrm{H}$ case is double the amount of that observed for the $\mathrm{H} 2 \mathrm{H}$ case. This may be related to the number of wrinkle branches interacting or merging at the centre of the film. For the $4 \mathrm{H}$ case, there are eight branches interacting (two for each hole) while for the $\mathrm{H} 2 \mathrm{H}$ case there are only four branches.

Figures 6(c-e) show the out-of-plane displacement $z$ at transverse planes for different holes sizes for the 2-hole cases (c) with the original hole diameter $D(\mathrm{H} 2 \mathrm{H})$, (d) with 0.75 times $D$ (H2HS), and (e) with 1.25 times $D(\mathrm{H} 2 \mathrm{HL})$. It can be seen that the wrinkling profile of the H2HS case is very similar to that of the $\mathrm{H} 2 \mathrm{H}$ case showing a well-defined anti-symmetry configuration. When compared to the $\mathrm{H} 2 \mathrm{H}$ case, the H2HS case shows smaller displacement amplitude for a given strain. These observations show that the reduction of the hole diameter in the H2HS case resulted in less interactive wrinkle branches when compared to the $\mathrm{H} 2 \mathrm{H}$ case, which in turn produced a better defined wrinkling profile with smaller displacement amplitude. The case with larger hole diameter H2HL resulted in a large single peak with a displacement amplitude ten times larger than that of the H2HS case and five times larger than that of the $\mathrm{H} 2 \mathrm{H}$ case. This peak has a symmetry configuration, which is in contrast to the anti-symmetry configuration of the $\mathrm{H} 2 \mathrm{H}$ and $\mathrm{H} 2 \mathrm{HS}$ cases. This could be explained by a strong wrinkles interaction in the mid-section due to the increase of the hole diameter.

\subsection{Evolutions of wavelength and amplitude of wrinkling patterns}

For all geometries considered in previous sections, we now calculate the evolutions of the mean wavelength and amplitude of the wrinkles in the mid-section at different loading stages (Fig. 7). Our calculated wavelength and amplitude are compared with the scaling laws developed for stretched elastic films (blue solid lines in Fig. 7) proposed by Cerda and Mehadevan [1] as $\lambda=(2 \pi L t)^{1 / 2} /$ $\left[3\left(1-v^{2}\right) \varepsilon\right]^{1 / 4}$ and $A=(v L t)^{1 / 2}\left[16 \varepsilon / 3 \pi^{2}\left(1-v^{2}\right)\right]^{1 / 4}$, respectively, with the sample length $L=1600 \mu \mathrm{m}$ and film thickness $t=0.2 \mu \mathrm{m}$. The corresponding slopes of the elasto-plastic cases in 
the log-log plots are the exponents of the power laws, which are clearly different from the elastic cases (as $-1 / 4$ and $1 / 4$ for the wavelength and amplitude, respectively). The surprising finding is the invariance of the mean wavelength for the elasto-plastic cases, irrespective to the wrinkling interaction, which is shown in Fig. 7(a). The wavelength evolution shows that as soon as the wrinkle profile is stable ( $\varepsilon>\sim 0.05 \%$, beyond the unstable loading-displacement response region), the mean wavelength does not change significantly during further loading. Note that the wavelength was calculated for only those significantly wrinkled regions, i.e., the wrinkled amplitude is more than 5\% of the maximum amplitude. In some cases (H2H, H2HS), the wavelength decreases with increasing strain when $\varepsilon>\sim 0.6 \%$. This could be explained by zones with large localised plastic strain in these cases. For the mean amplitude evolution shown in Fig. 7(b), an almost linear trend is seen (power of 1.0 , instead of $1 / 4$ for elastic cases) for the $4 \mathrm{H}, \mathrm{H} 2 \mathrm{H}, \mathrm{H} 2 \mathrm{HL}, \mathrm{H} 2 \mathrm{HS}, \mathrm{A} 2 \mathrm{H}, \mathrm{H} 2 \mathrm{H} 2 \mathrm{X}$ cases in which strong interacting wrinkles developed. For the $\mathrm{H} 2 \mathrm{H}$ case spaced by three times the original distance $(\mathrm{H} 2 \mathrm{H} 3 \mathrm{X})$ with less wrinkling interaction, the amplitude is significant smaller and the power is reduced to almost $1 / 4$, which is comparable with the elastic case. These findings suggest that stable wrinkling patterns can be developed on elasto-plastic films, and the mean wavelength is insensitive to the loading before large plastic deformation occurs. Meanwhile, the wrinkling amplitude can be tuned sensitively through applied stretch. Both are important for designing the desired wrinkling structures.

When comparing the evolving wrinkling patterns from different pre-patterned geometries, we conclude that interacting wrinkles originating from defects play a crucial role in the resulting wrinkling pattern. The geometrical parameters investigated here, such as number, size and distribution of these defects and the spacing between them, can be manipulated to determine the resulting wrinkling patterns and branches. For example, if one is looking for applications requiring smooth profiles but small amplitudes, our results suggest that the weakly-interacting wrinkles cases lead to smaller wrinkling amplitudes, yet may provide a smoother wrinkling pattern when compared to the non-interacting case. For applications that require smooth wrinkling patterns with large amplitudes, two holes that are closely spaced are lead to this type of wrinkling. The results also suggest that higher wrinkling frequency (4-hole case) comes at the expense of a well-defined wrinkle shape. This indicates that judicious patterning and engineering of periodic arrays of defects may lead to more well-defined wrinkling patterns in such materials. The understanding of wrinkling evolution and interactions brought about by this study could guide the development of wrinkle "microstructures" with controlled wrinkle domains useful for a range of structural and functional applications. Such pattern features include symmetric or anti-symmetric domains with controlled wavelengths and amplitudes. The numerical simulations presented here provide an initial step towards connecting the evolving and interacting wrinkling patterns under different stress-strain conditions in Al thin films due to pre-patterned defects.

\section{Conclusions}

In this work, we simulated the wrinkling patterns formed from intentionally-defected freestanding elasto-plastic metal thin films under tensile loading. The defects were introduced via an array of ordered microholes in different configurations. We investigated the effect of hole spacing on wrinkling patterns for horizontal 2-hole geometries followed by different spatial configurations of holes and defect size. In addition to the validation of resulting wrinkling patterns with micro-tensile experiments of $\mathrm{Al}$ thin films, the numerical simulations predicted taut, wrinkled and slack regions of the stretched film with detailed stress-strain profiles. Our results show that plasticity plays an important role in development of wrinkling patterns and their interactions in Al thin films, exhibiting different evolutions of wrinkle wavelength and amplitude during loading as compared to existing scaling laws for stretched elastic films. These simulations could be extended to quantify residual stresses in constrained thin films with defects by analysing the wrinkling patterns that develop.

We found that spacing of defects controls the level of interaction between wrinkle branches generated at the defects. Strong interactions resulted in well-defined out-of-plane displacement profiles. Strong interactions also resulted in the merging of different wrinkle branches into one single wrinkle with large displacement amplitude in the out-of-plane direction. The interaction and merging of wrinkle patterns from multiple defects can be predicted using our numerical simulations with high 
confidence, suggesting further detailed studies on controlling and optimising the wrinkle structures for structural and functional applications by designing the defect geometries and configurations.

\section{Acknowledgements}

Financial support for this research from the Australian Research Council through Grant No. DE130101639 is gratefully appreciated. T.J.R. gratefully acknowledges support from the National Science Foundation through a CAREER Award No. DMR-1255305. D.S.G. and K.J.H acknowledge support by the U.S. Department of Energy, Office of Basic Energy Sciences, Division of Materials Science and Engineering under Awards \#DESC0008135 and \#DE-FG02-07ER46437.

\section{References}

[1] E. Cerda, L. Mahadevan, Geometry and physics of wrinkling, Phys. Rev. Lett. 90 (2003) 074302.

[2] R. Massabò, L. Gambarotta, Wrinkling of Plane Isotropic Biological Membranes, J. Appl. Mech 74 (2006) 550-559.

[3] J.Y. Chung, A.J. Nolte, C.M. Stafford, Surface Wrinkling: A Versatile Platform for Measuring Thin-Film Properties, Adv. Mater. 23 (2011) 349-368.

[4] H.X. Mei, R. Huang, J.Y. Chung, C.M. Stafford, H.H. Yu, Buckling modes of elastic thin films on elastic substrates, Appl. Phys. Lett. 90 (2007) 151902.

[5] W.T.S. Huck, N. Bowden, P. Onck, T. Pardoen, J.W. Hutchinson, G.M. Whitesides, Ordering of spontaneously formed buckles on planar surfaces, Langmuir 16 (2000) 3497-3501.

[6] K. Efimenko, M. Rackaitis, E. Manias, A. Vaziri, L. Mahadevan, J. Genzer, Nested self-similar wrinkling patterns in skins, Nat. Mater. 4 (2005) 293-297.

[7] H. Vandeparre, J. Leopoldes, C. Poulard, S. Desprez, G. Derue, C. Gay, P. Damman, Slippery or sticky boundary conditions: Control of wrinkling in metal-capped thin polymer films by selective adhesion to substrates, Phys. Rev. Lett. 99 (2007) 188302.

[8] C. Harrison, C.M. Stafford, W.H. Zhang, A. Karim, Sinusoidal phase grating created by a tunably buckled surface, Appl. Phys. Lett. 85 (2004) 4016-4018.

[9] C.M. Stafford, C. Harrison, K.L. Beers, A. Karim, E.J. Amis, M.R. Vanlandingham, H.C. Kim, W. Volksen, R.D. Miller, E.E. Simonyi, A buckling-based metrology for measuring the elastic moduli of polymeric thin films, Nat. Mater. 3 (2004) 545-550.

[10] W.Z. Bao, F. Miao, Z. Chen, H. Zhang, W.Y. Jang, C. Dames, C.N. Lau, Controlled ripple texturing of suspended graphene and ultrathin graphite membranes, Nat. Nanotechnol. 4 (2009) 562-566.

[11] J. Huang, M. Juszkiewicz, W.H. de Jeu, E. Cerda, T. Emrick, N. Menon, T.P. Russell, Capillary wrinkling of floating thin polymer films, Science 317 (2007) 650-653.

[12] X.M. Lu, Y.N. Xia, Electronic materials - Buckling down for flexible electronics, Nat. Nanotechnol. 1 (2006) 163-164.

[13] M. Takahashi, T. Maeda, K. Uemura, J.X. Yao, Y.M. Tokuda, T. Yoko, H. Kaji, A. Marcelli, P. Innocenzi, Photoinduced formation of wrinkled microstructures with long-range order in thin oxide films, Adv. Mater. 19 (2007) $4343-4346$.

[14] A. Tessler, D.W. Sleight, Geometrically nonlinear shell analysis of wrinkled thin-film membranes with stress concentrations, J. Spacecraft Rockets 44 (2007) 582-588.

[15] E.A. Flores-Johnson, L. Shen, R.K. Annabattula, P.R. Onck, Y.G. Shen, Z. Chen, The effect of interface adhesion on buckling and cracking of hard thin films, Appl. Phys. Lett. 105 (2014) 161912.

[16] E.P. Chan, A.J. Crosby, Fabricating microlens arrays by surface wrinkling, Adv. Mater. 18 (2006) 3238-3242.

[17] M.W. Moon, S.H. Lee, J.Y. Sun, K.H. Oh, A. Vaziri, J.W. Hutchinson, Wrinkled hard skins on polymers created by focused ion beam, Proc. Natl. Acad. Sci. USA 104 (2007) 1130-1133.

[18] P.C. Lin, S. Vajpayee, A. Jagota, C.Y. Hui, S. Yang, Mechanically tunable dry adhesive from wrinkled elastomers, Soft Matter 4 (2008) 1830-1835.

[19] A. Mathur, J. Erlebacher, Size dependence of effective Young's modulus of nanoporous gold, Appl. Phys. Lett. 90 (2007) 061910.

[20] Y.F. Mei, S. Kiravittaya, S. Harazim, O.G. Schmidt, Principles and applications of micro and nanoscale wrinkles, Mater. Sci. Eng., R 70 (2010) 209-224.

[21] Y. Wong, S. Pellegrino, K. Park, Prediction of Winkle Amplitudes in Square Solar Sails, in 44th AIAA/ASME/ASCE/AHS/ASC Structures, Structural Dynamics, and Materials Conference 2003, American Institute of Aeronautics and Astronautics.

[22] J. Genzer, J. Groenewold, Soft matter with hard skin: From skin wrinkles to templating and material characterization, Soft Matter 2 (2006) 310-323.

[23] C.Q. Ru, Surface wrinkling of two mutually attracting elastic thin films due to van der Waals forces, J. Appl. Phys. 90 (2001) 6098-6104.

[24] H. Vandeparre, S. Gabriele, F. Brau, C. Gay, K.K. Parker, P. Damman, Hierarchical wrinkling patterns, Soft Matter 6 (2010) 5751-5756. 
[25] Y. Zhao, W.M. Huang, Y.Q. Fu, Formation of micro/nano-scale wrinkling patterns atop shape memory polymers, J. Micromech. Microeng. 21 (2011) 067007.

[26] P. Kim, M. Abkarian, H.A. Stone, Hierarchical folding of elastic membranes under biaxial compressive stress, Nat. Mater. 10 (2011) 952-957.

[27] A.M. Clausen, D.M. Paskiewicz, A. Sadeghirad, J. Jakes, D.E. Savage, D.S. Stone, F. Liu, M.G. Lagally, Silicon nanomembranes as a means to evaluate stress evolution in deposited thin films, Extreme Mech. Lett. 1 (2014) 9-16.

[28] D. Yan, K. Zhang, F. Peng, G. Hu, Tailoring the wrinkle pattern of a microstructured membrane, Appl. Phys. Lett. 105 (2014) 071905.

[29] F. Andreussi, M.E. Gurtin, On the wrinkling of a free surface, J. Appl. Phys. 48 (1977) 3798-3799.

[30] A.K. Ghosh, Tensile instability and necking in materials with strain hardening and strain-rate hardening, Acta Metall. 25 (1977) 1413-1424.

[31] J.W. Hutchinson, K.W. Neale, Influence of Strain-Rate Sensitivity on Necking under Uniaxial Tension, Acta Metall. 25 (1977) 839-846.

[32] M. Lewicka, L. Mahadevan, M.R. Pakzad, The Föppl-von Kármán equations for plates with incompatible strains, Proc. R. Soc. A 467 (2011) 402-426.

[33] Y.M. Wang, E. Ma, Strain hardening, strain rate sensitivity, and ductility of nanostructured metals, Mater. Sci. Eng., A 375 (2004) 46-52.

[34] H.M. Lee, S.-Y. Choi, K.T. Kim, J.-Y. Yun, D.S. Jung, S.B. Park, J. Park, A Novel Solution-Stamping Process for Preparation of a Highly Conductive Aluminum Thin Film, Adv. Mater. 23 (2011) 5524-5528.

[35] SIMULIA, Abaqus Analysis User's Manual (Version 6.12), (2012)

[36] R.K. Annabattula, W.T.S. Huck, P.R. Onck, Micron-scale channel formation by the release and bond-back of prestressed thin films: A finite element analysis, J. Mech. Phys. Solids 58 (2010) 447-465.

[37] J.A. Floro, S.J. Hearne, J.A. Hunter, P. Kotula, E. Chason, S.C. Seel, C.V. Thompson, The dynamic competition between stress generation and relaxation mechanisms during coalescence of Volmer-Weber thin films, J. Appl. Phys. 89 (2001) 4886-4897.

[38] D.S. Gianola, S. Van Petegem, M. Legros, S. Brandstetter, H. Van Swygenhoven, K.J. Hemker, Stress-assisted discontinuous grain growth and its effect on the deformation behavior of nanocrystalline aluminum thin films, Acta Mater. 54 (2006) 2253-2263.

[39] E. Riks, The Application of Newton's Method to the Problem of Elastic Stability, J. Appl. Mech. 39 (1972) 1060-1065.

[40] E. Riks, An incremental approach to the solution of snapping and buckling problems, Int. J. Solids Struct. 15 (1979) 529-551.

[41] T.J. Rupert, D.S. Gianola, Y. Gan, K.J. Hemker, Experimental Observations of Stress-Driven Grain Boundary Migration, Science 326 (2009) 1686-1690.

[42] M. Taylor, K. Bertoldi, D.J. Steigmann, Spatial resolution of wrinkle patterns in thin elastic sheets at finite strain, J. Mech. Phys. Solids 62 (2014) 163-180.

[43] B. Tabarrok, Z. Qin, Nonlinear analysis of tension structures, Comput. Struct. 45 (1992) 973-984.

[44] V. Nayyar, K. Ravi-Chandar, R. Huang, Stretch-induced stress patterns and wrinkles in hyperelastic thin sheets, Int. J. Solids Struct. 48 (2011) 3471-3483. 


\section{Figure captions}

Figure 1. Submicron freestanding patterned Al thin films. (a) A typical finite element mesh for 4-hole patterned thin film, with a subfigure showing the refined mesh near one patterned hole. (b) Schematic of microfabricated testing frame. Testing geometries of (c) horizontal 2-hole $(\mathrm{H} 2 \mathrm{H})$, (d) angled 2-hole $(\mathrm{A} 2 \mathrm{H})$, and $(\mathrm{e}) 4$-hole $(4 \mathrm{H})$ pattern to introduce spatial variations of stress and strain.

Figure 2. Simulations of the effect of plasticity on wrinkling patterns for horizontal 2-hole $(\mathrm{H} 2 \mathrm{H})$ geometry: (a) elastic-plastic, (b) elastic-perfectly plastic, (c) elastic (the left subfigures in (a)-(c) are the actual out-of-plane deformation profiles at the transverse midplane). Depicting effect of lighting orientation on wrinkling-induced contrast in $\mathrm{H} 2 \mathrm{H}$ geometry: apparent simulated wrinkling patterns for (d) normal and (e) angled incidence of lighting, demonstrating how light would be reflected, affecting subsequent interpretation of real wrinkling wavelength. In (d) and (e), the corresponding actual (blue) and apparent (yellow) out-of-plane deformation profiles are compared under the normal and angled lighting conditions.

Figure 3. Simulated (left columns) and experimental (right columns) wrinkling patterns during tensile testing of freestanding $\mathrm{Al}$ thin film: (a) horizontal 2-hole configuration $(\mathrm{H} 2 \mathrm{H})$, (b) angled 2-hole configuration $(\mathrm{A} 2 \mathrm{H})$, and (c) 4-hole configuration $(4 \mathrm{H})$. Bottom inset shows taut, wrinkled and slack regions (area delimited by the dashed red squares).

Figure 4. Contour plots of in-plane minimum principal stress $\sigma_{2}$ and equivalent plastic strain $\varepsilon_{e q}$ during simulations of tensile testing of hole-patterned $\mathrm{Al}$ thin films.

Figure 5. Contour plots of in-plane minimum principal stress $\sigma_{2}$ evolution during simulations of tensile testing of (a) 1-hole case $(1 \mathrm{H})$, (b) 2 holes separated by three times the original distance (separation in the $\mathrm{H} 2 \mathrm{H}$ case), (c) 2 holes separated by twice the original distance, (d) 2-hole case $(\mathrm{H} 2 \mathrm{H}),(\mathrm{e})$ Out-of-plane displacement $z$ along transverse planes.

Figure 6. Simulations of the out-of-plane displacement $z$ along the mid-section of the film under increasing nominal strain for different arrays of holes: (a) $4 \mathrm{H}$, (b) $\mathrm{A} 2 \mathrm{H}$, (c) $\mathrm{H} 2 \mathrm{H}$, (d) $\mathrm{H} 2 \mathrm{HS}$, (e) $\mathrm{H} 2 \mathrm{HL}$. The insets at the top show wrinkling patterns at $0.31 \%$ strain.

Figure 7. (a) Mean wavelength and (b) mean amplitude against strain for different arrangements of geometric defects. The blue solid lines correspond to the power-law relations developed for elastic films [1]. 
Figure 1
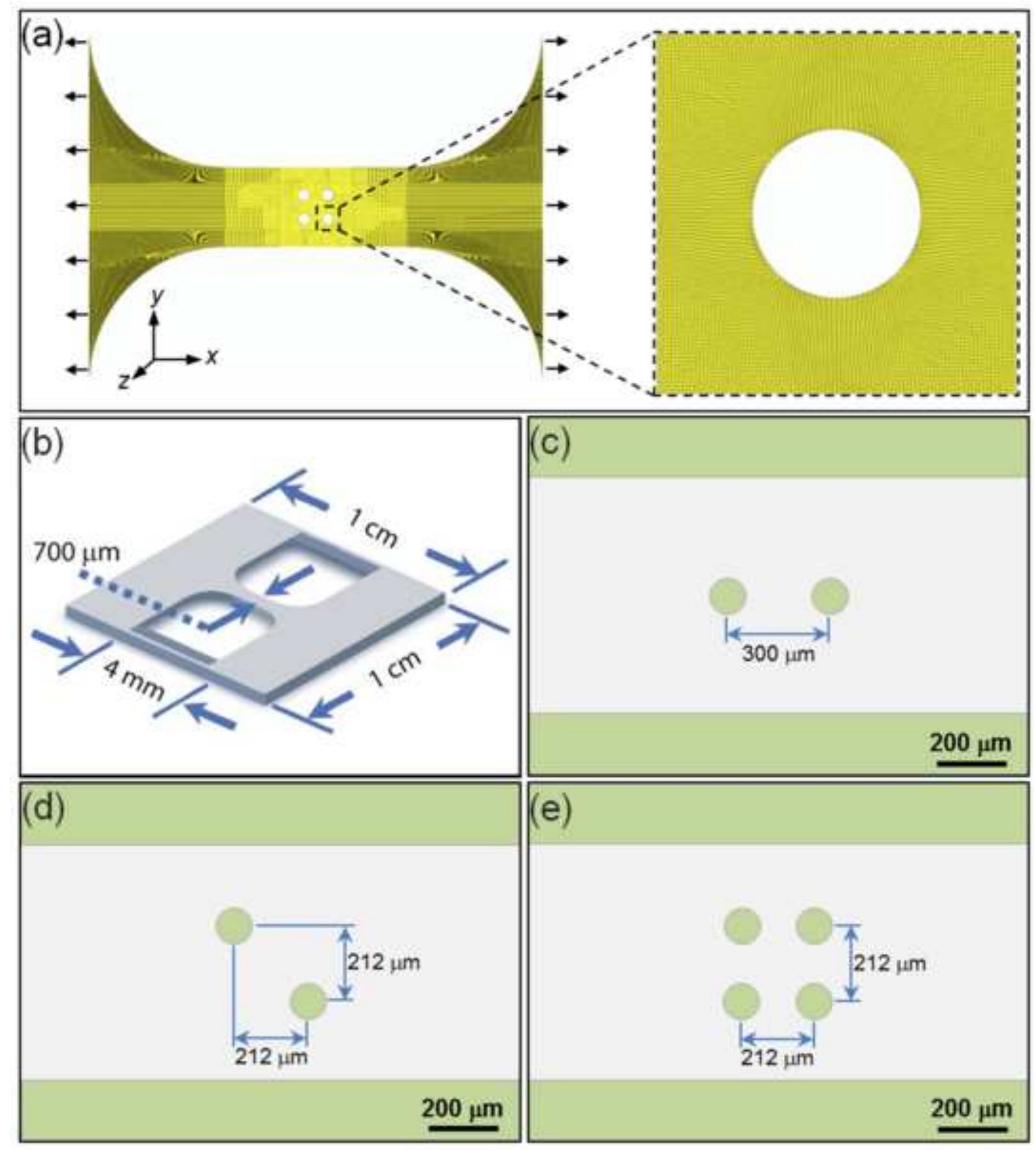
(a) Elastic-plastic
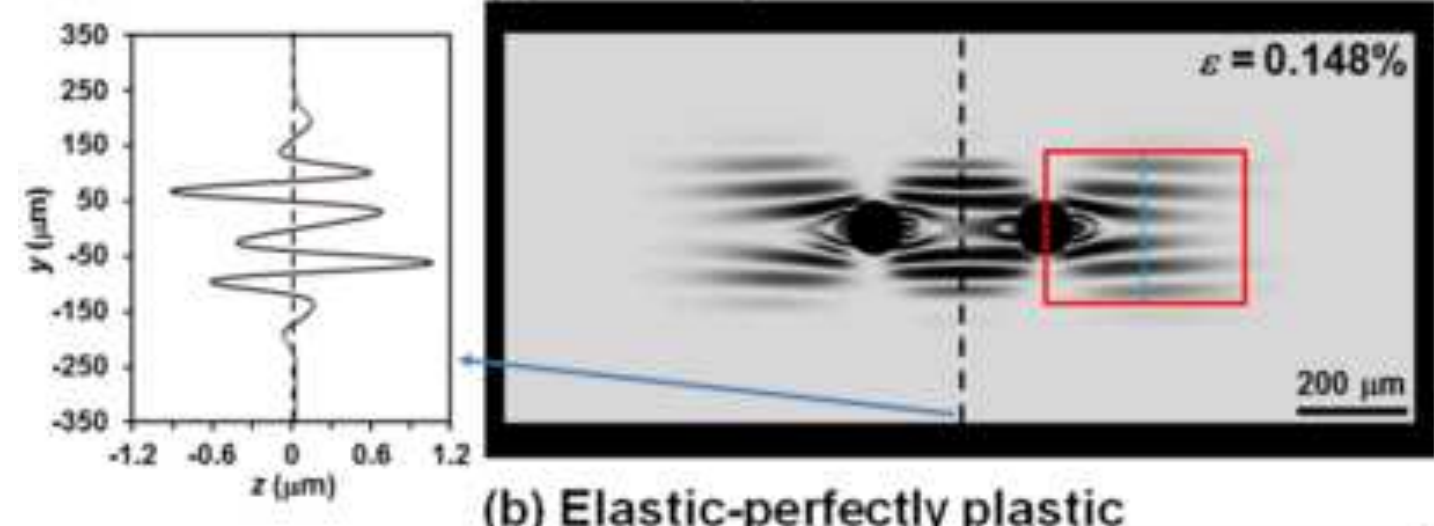

(b) Elastic-perfectly plastic
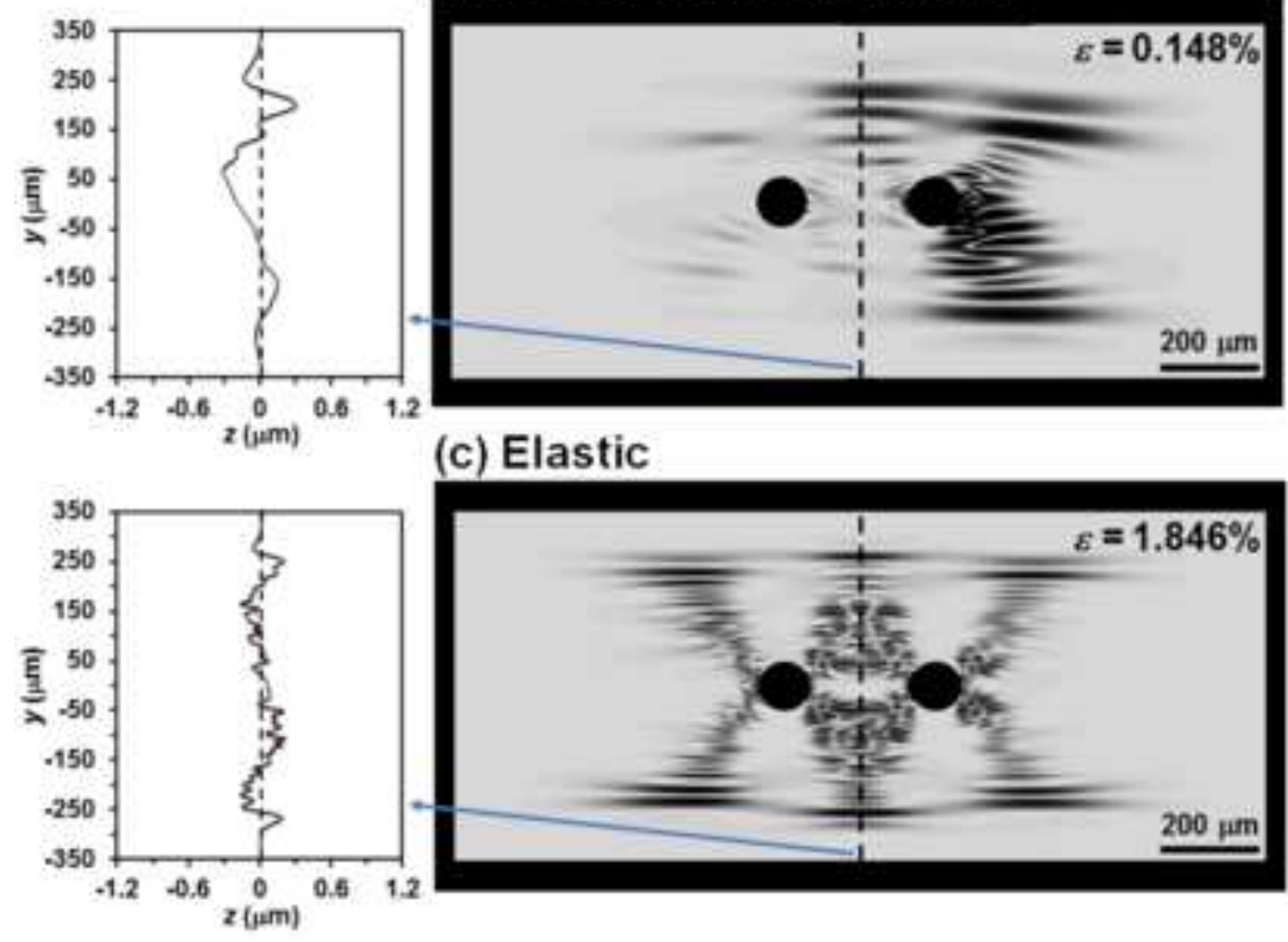

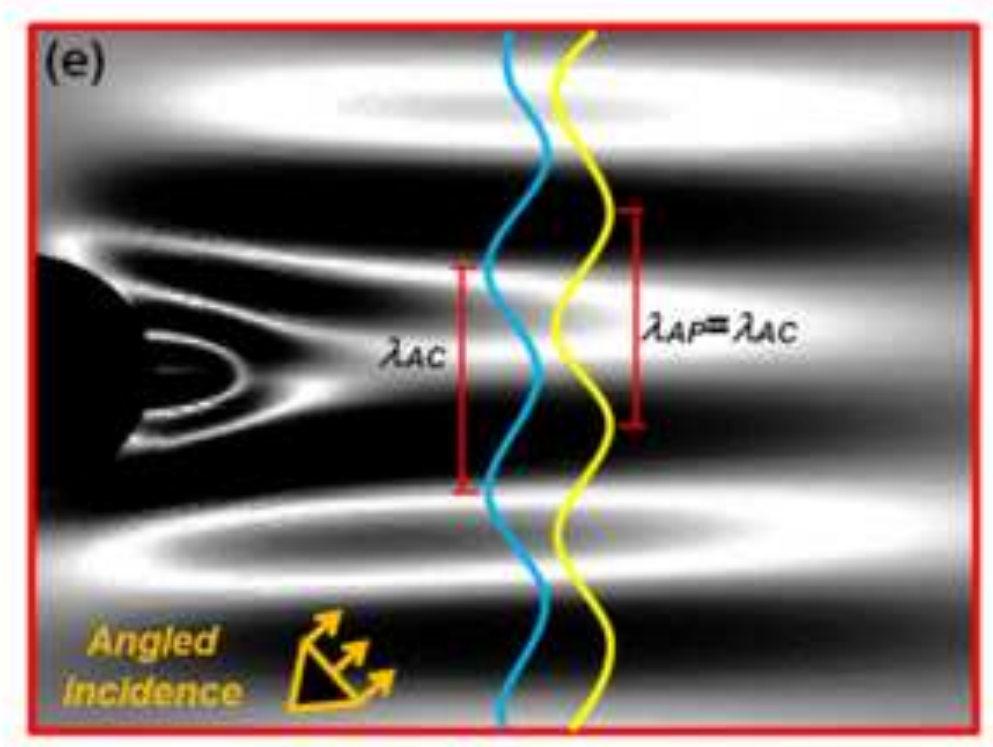


(a) Horizontal 2-hole array $(\mathrm{H} 2 \mathrm{H})$ Simulation

\section{1\%} .
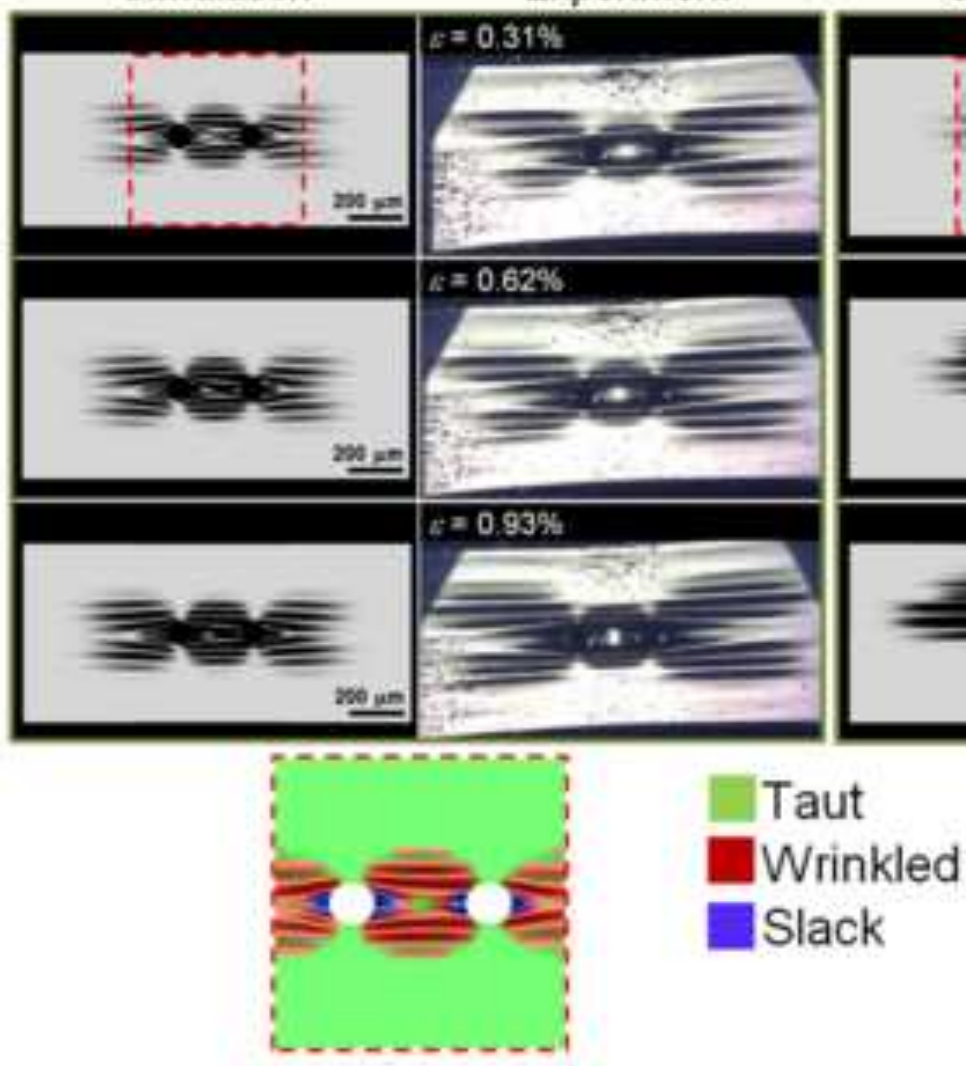

(b) Angled 2-hole array (A2H)

Simulation
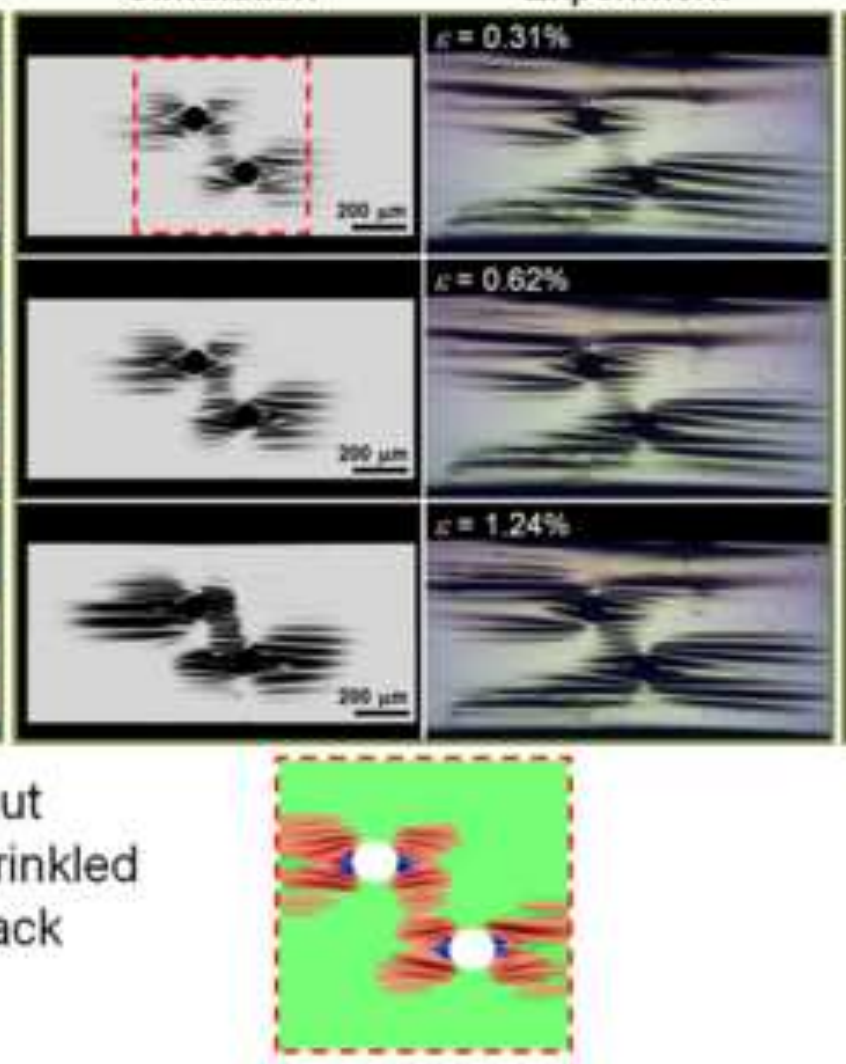

(c) 4-hole array $(4 \mathrm{H})$

Simulation
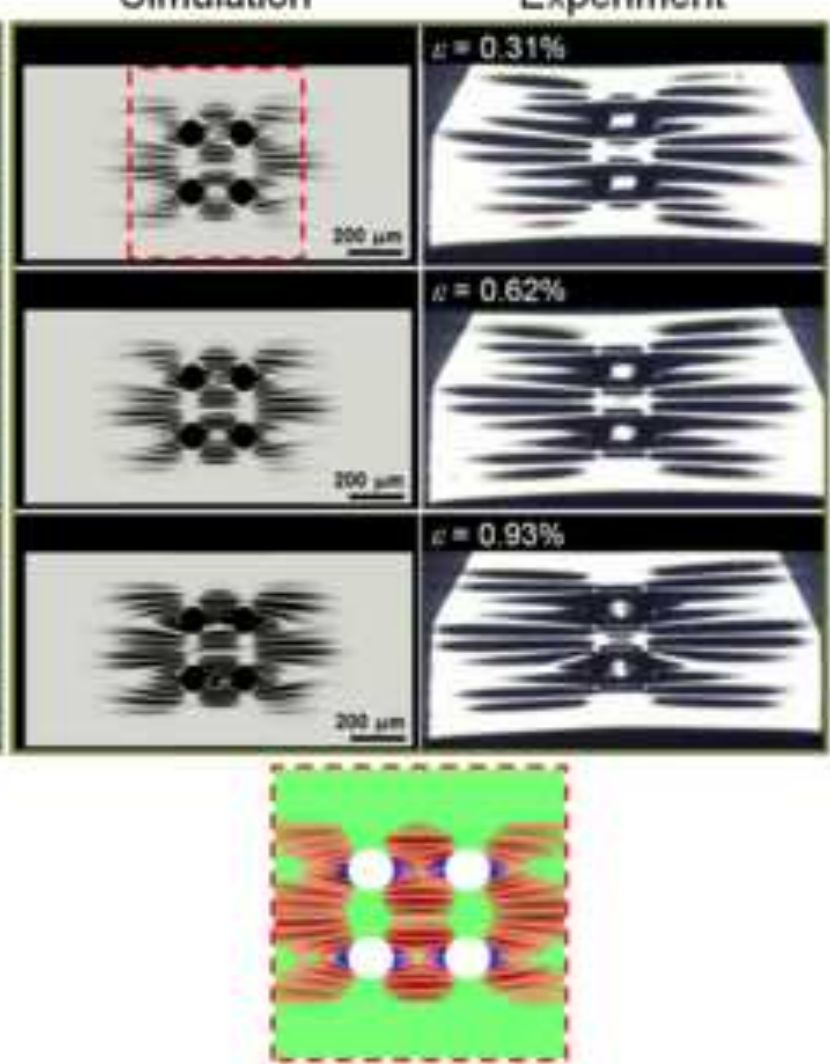

Taut

Wrinkled

Slack
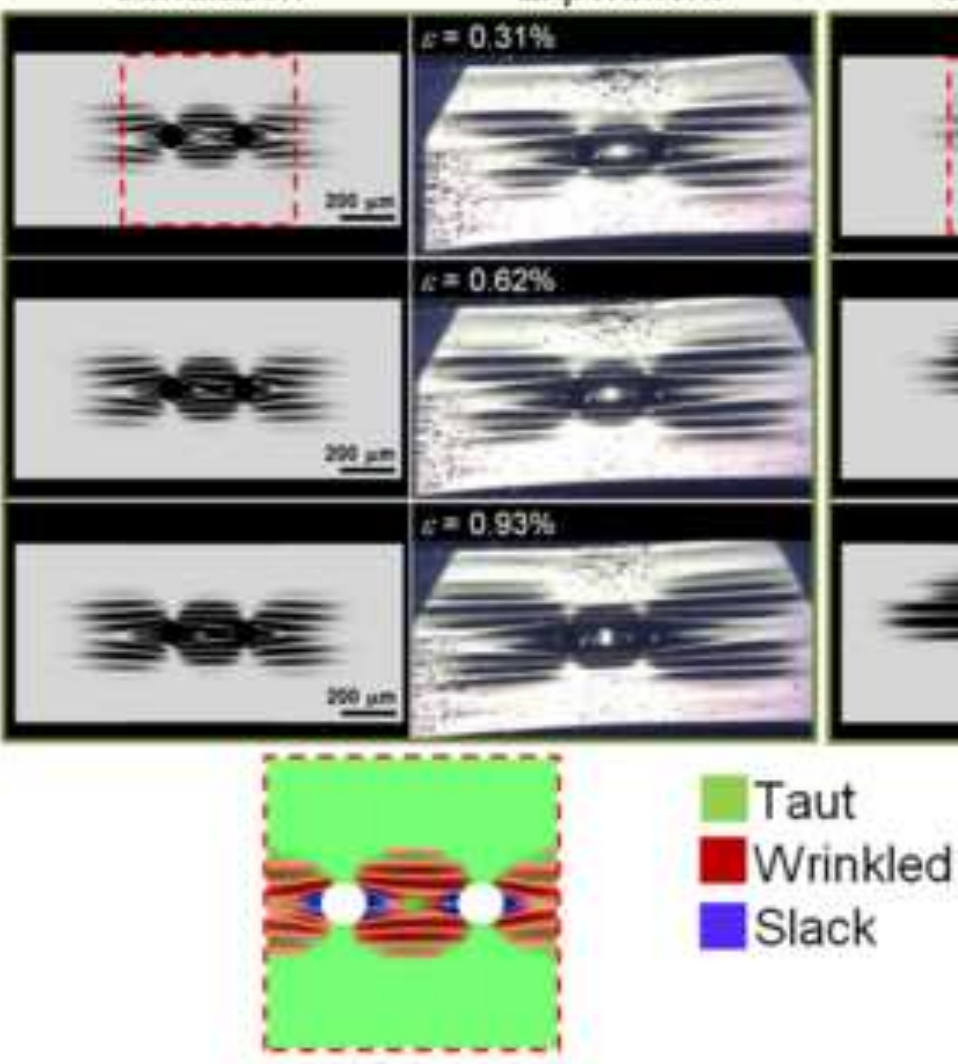
(a) $\mathrm{H} 2 \mathrm{H}$
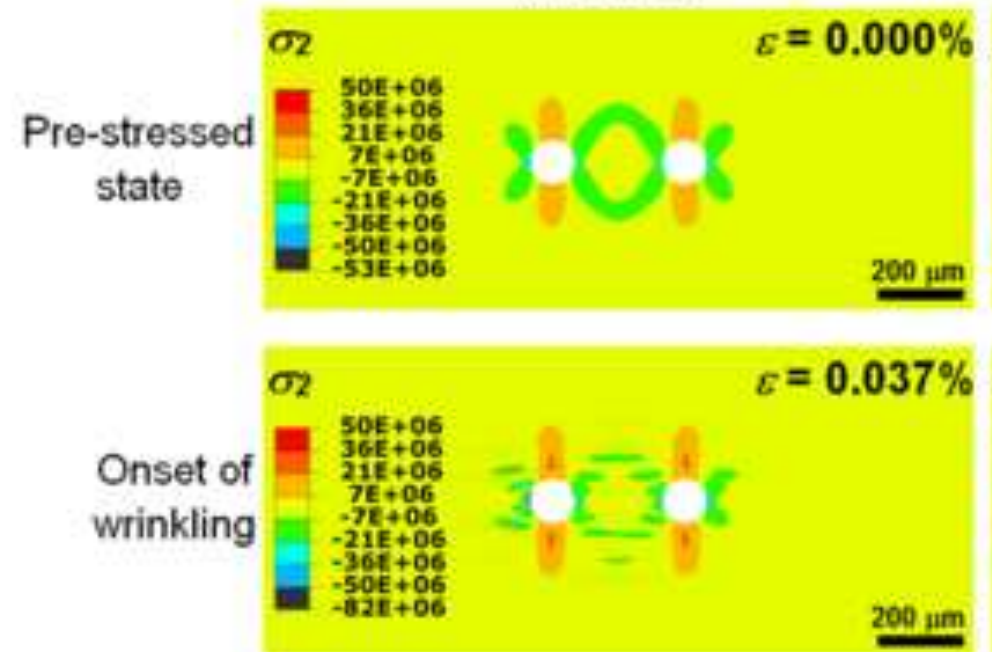

(b) $\mathrm{A} 2 \mathrm{H}$

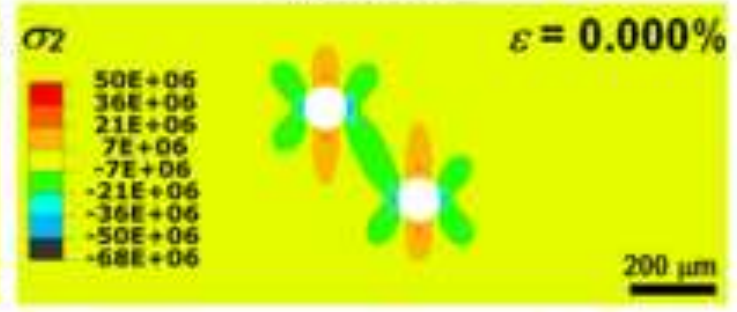

(c) $4 \mathrm{H}$

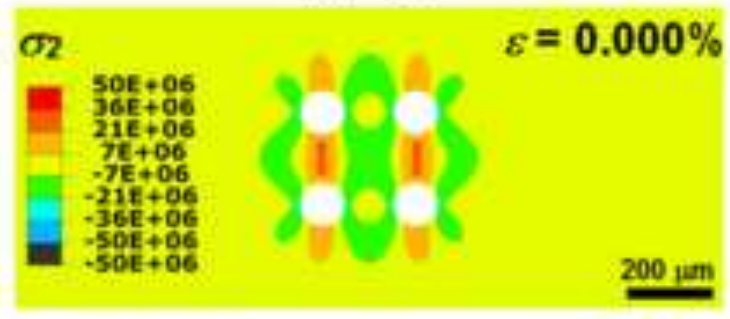

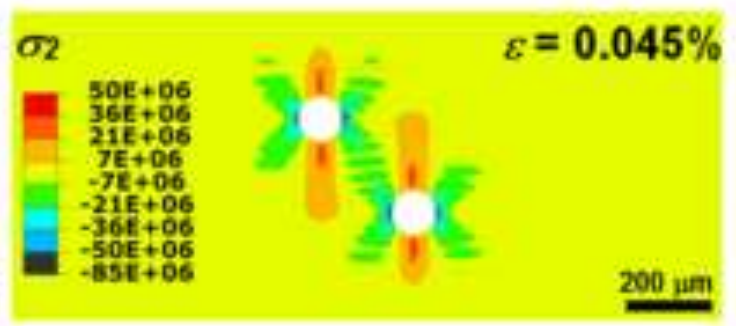

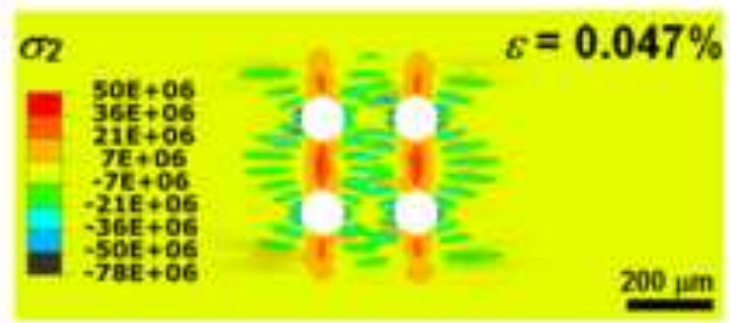

Strain at which
the out-of-plane
deformation
profile is stable

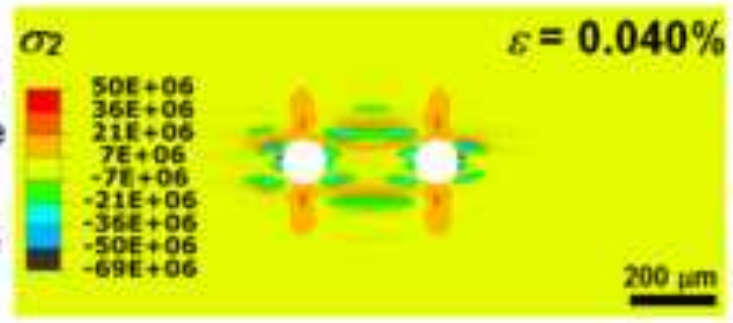

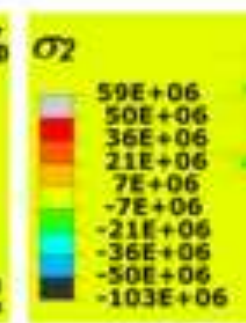
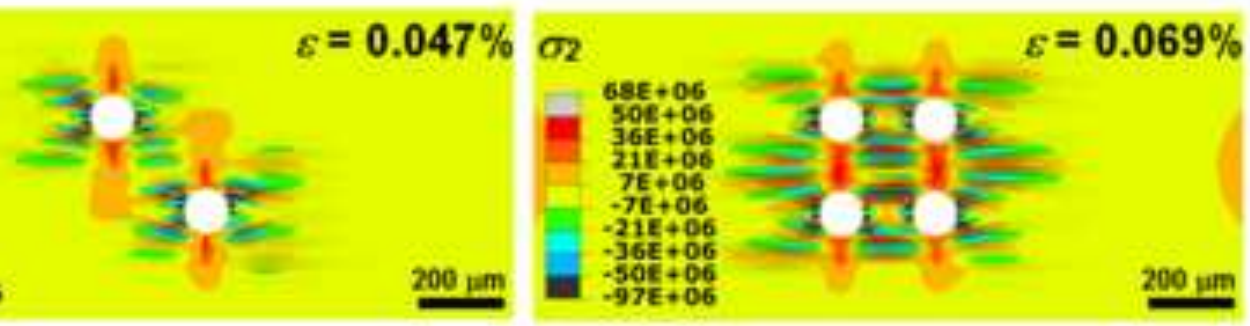

Contour plot
of $\sigma_{2}$ at
$\ell=0.31 \%$
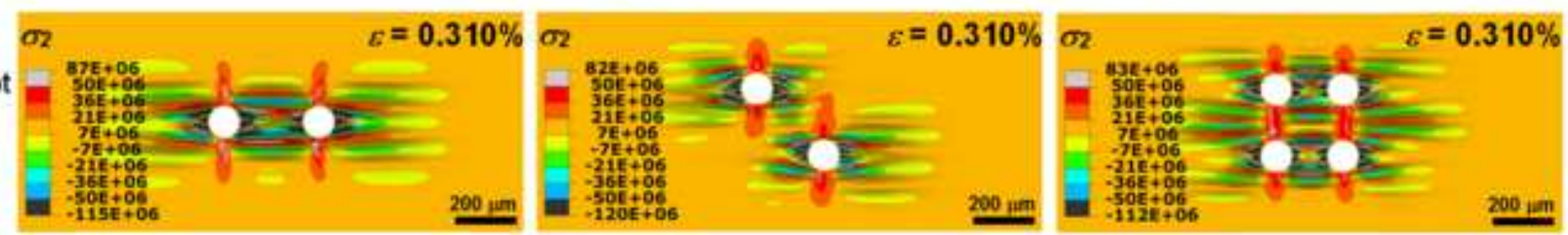

Eeq

$\varepsilon=0.310 \% \varepsilon$ eq
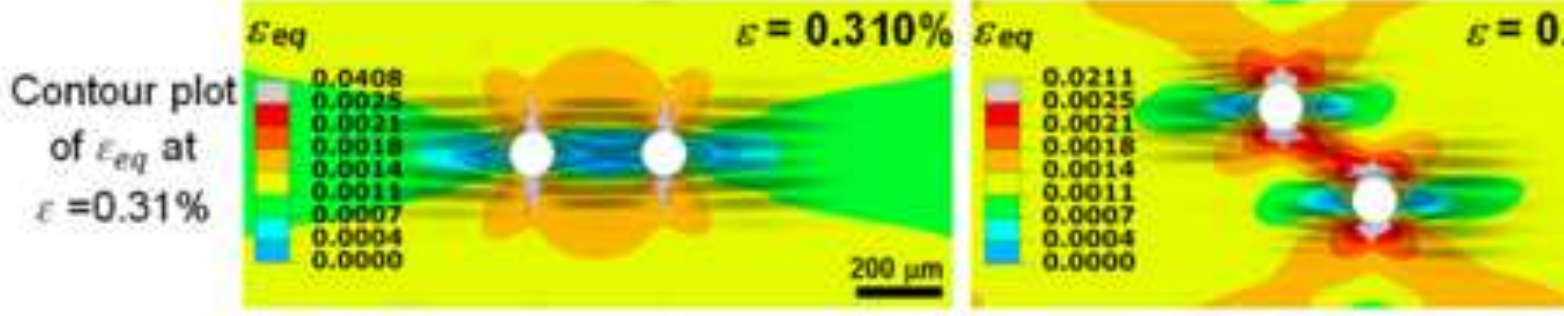

$200 \mathrm{~mm}$

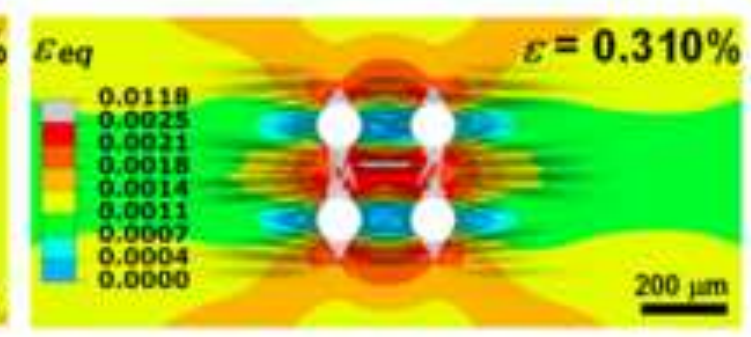


(a) $1 \mathrm{H}$

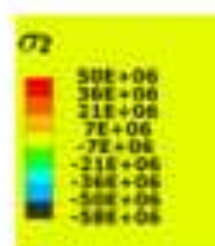

(b) 2 holes separated by three

times the original distance (c) 2 holes separated by twice the original distance (d) $\mathrm{H} 2 \mathrm{H}$

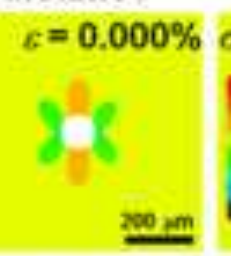

$\tau=0.000 \%$ or ant

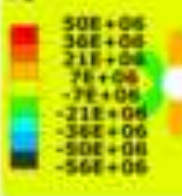

200.10

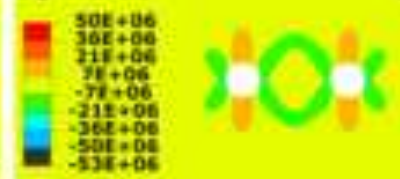

$\varepsilon=0.000 \%$
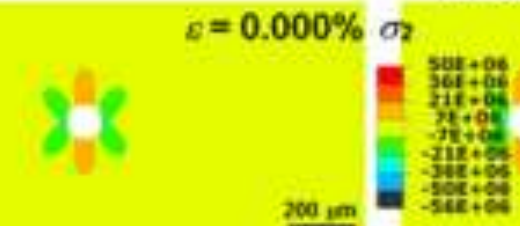

$051 \%$
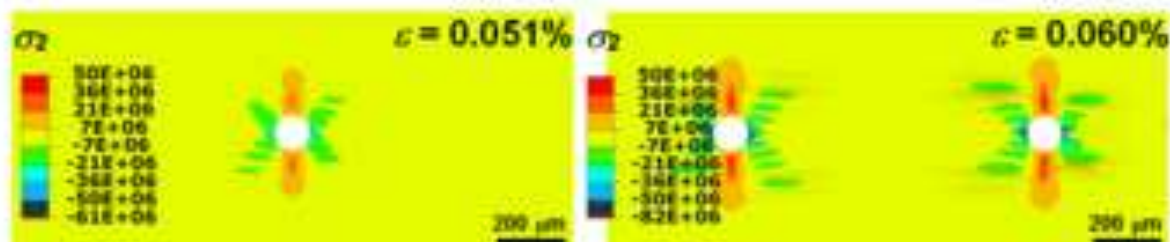

oㅡㄹ
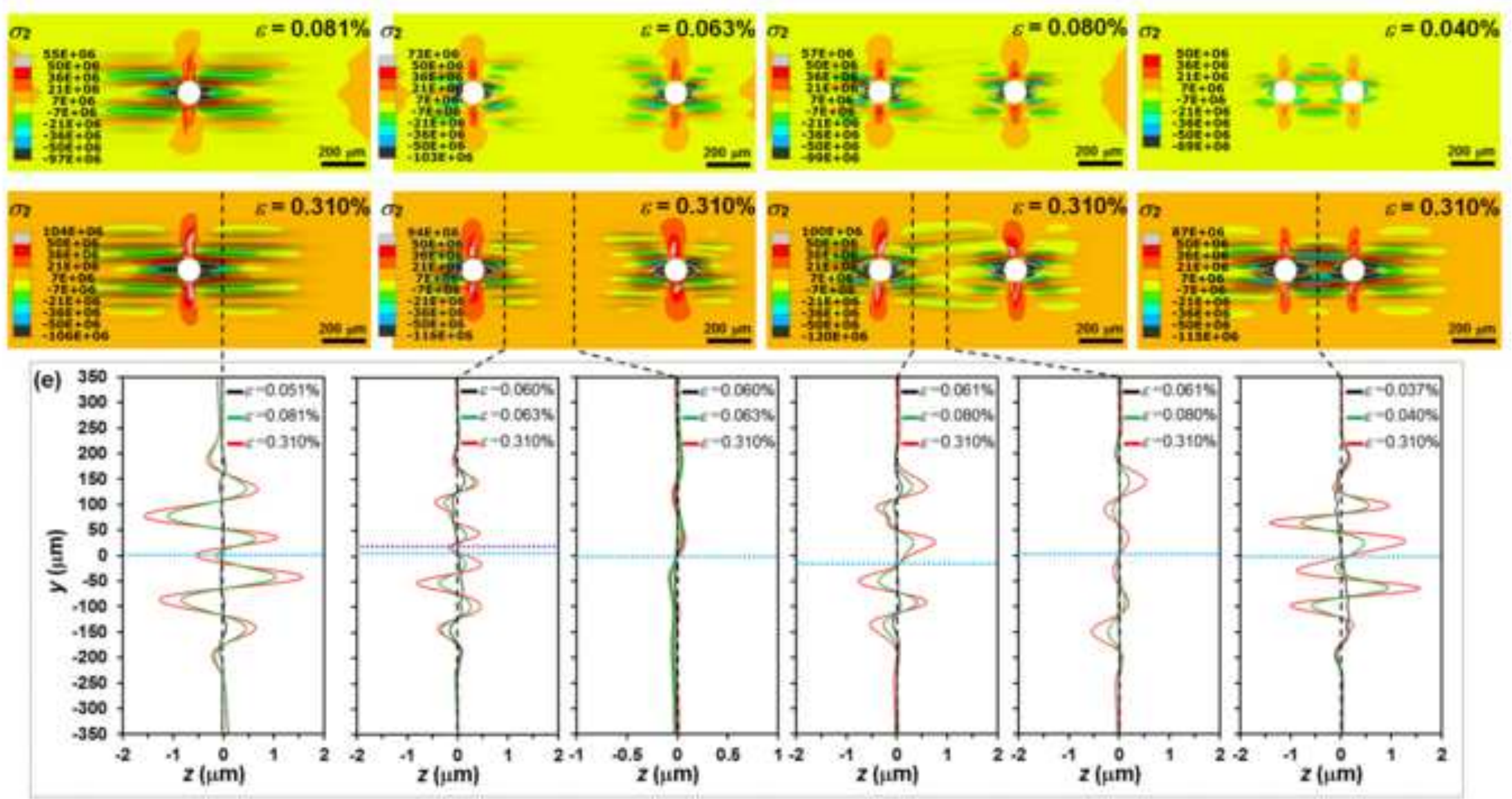

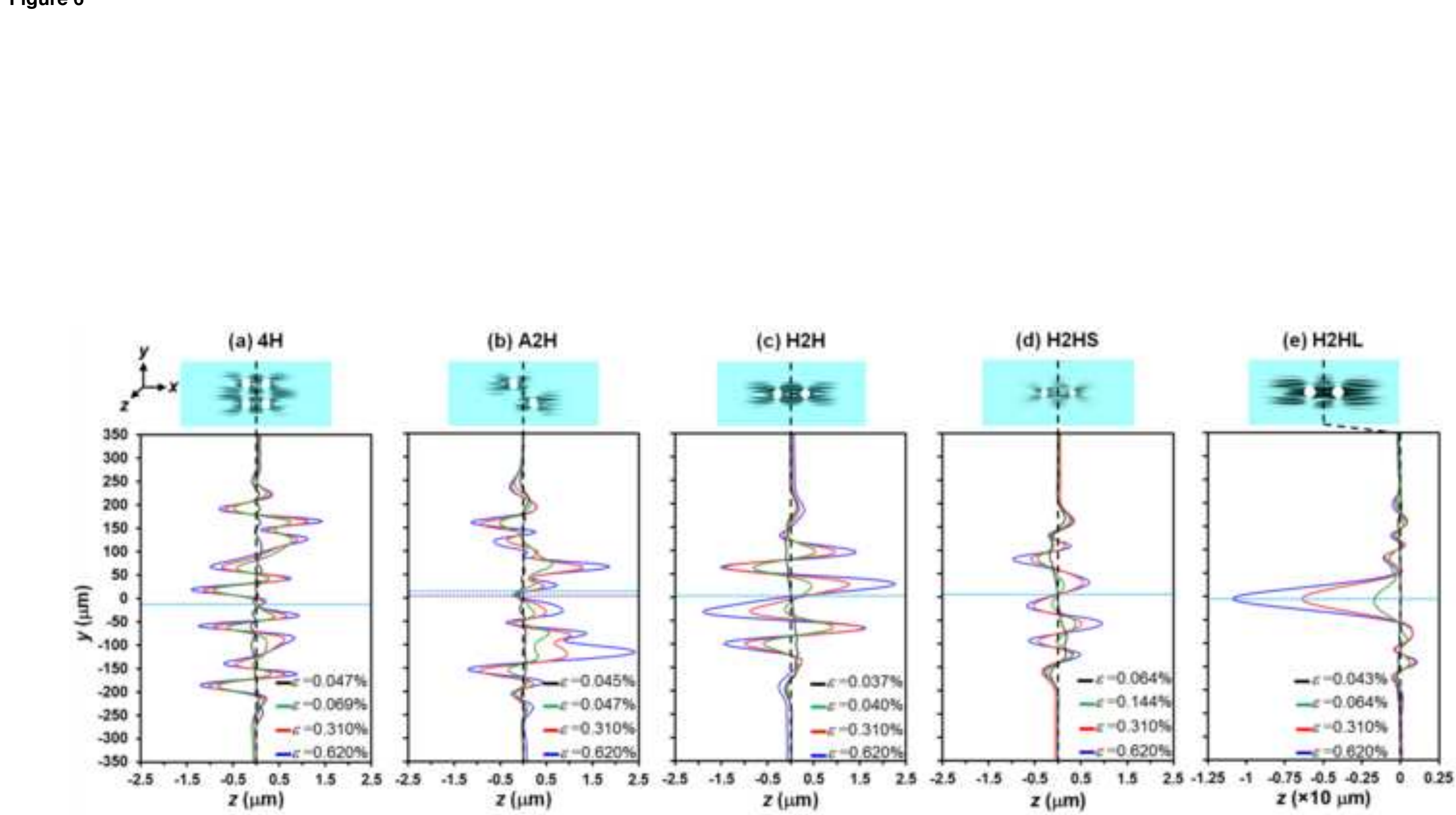
(a)

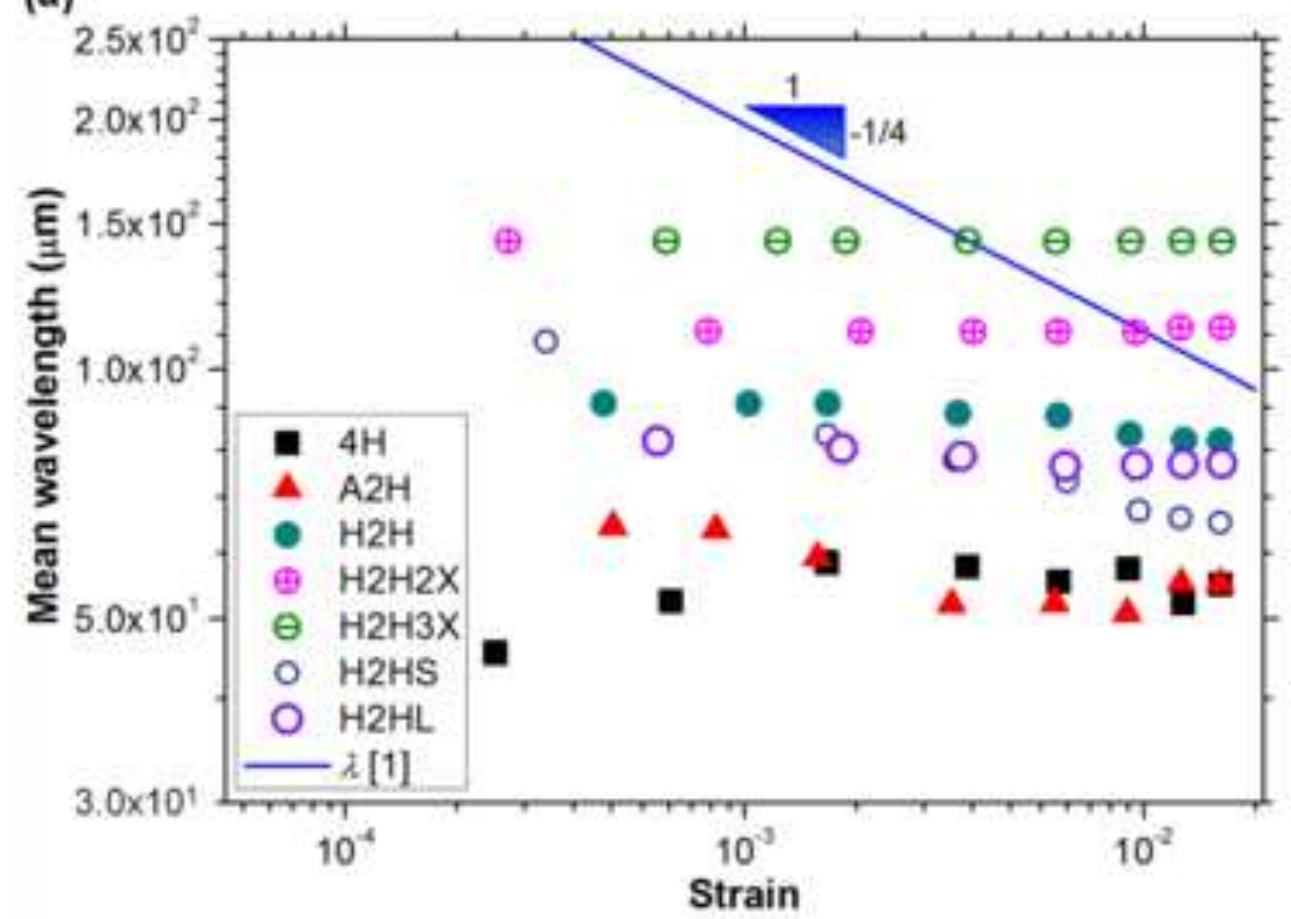

(b)

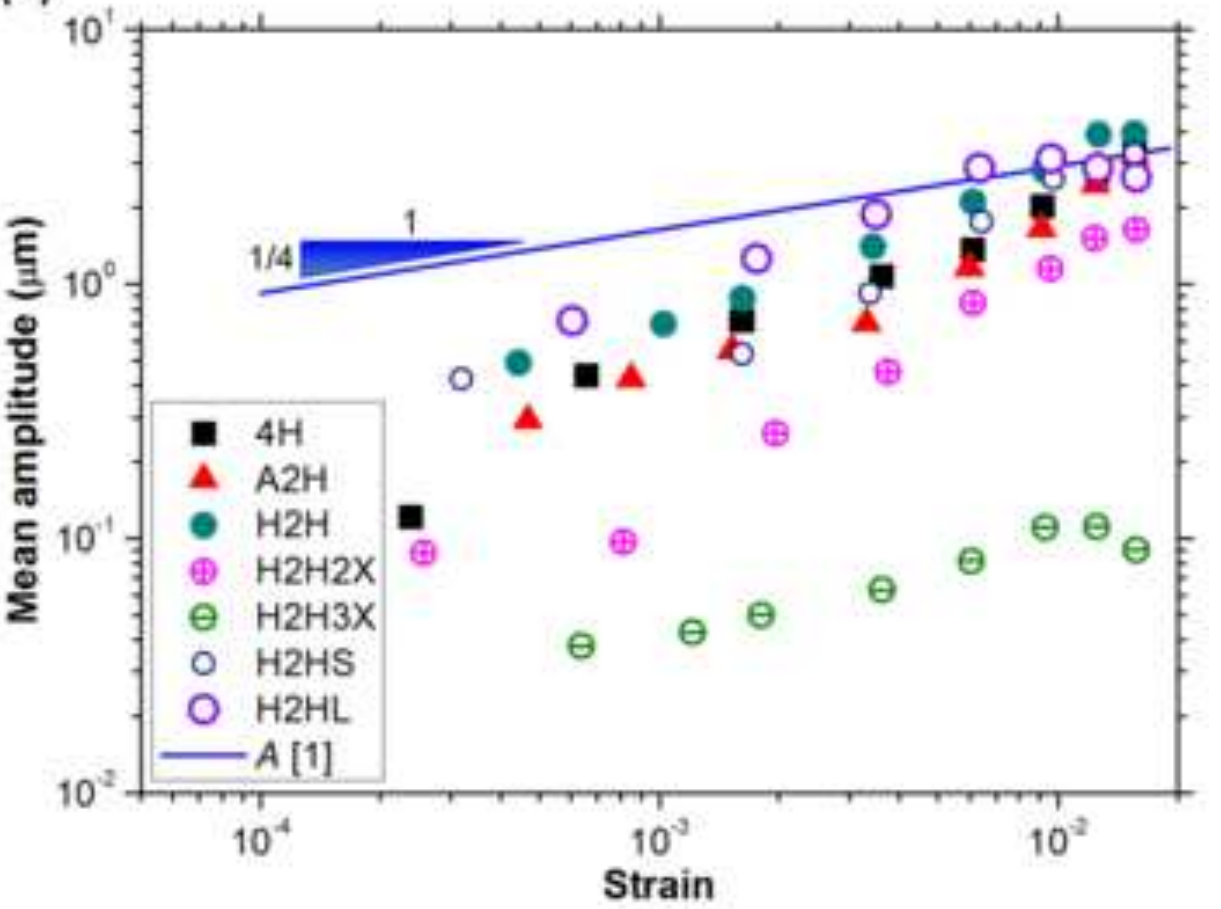


Experiment
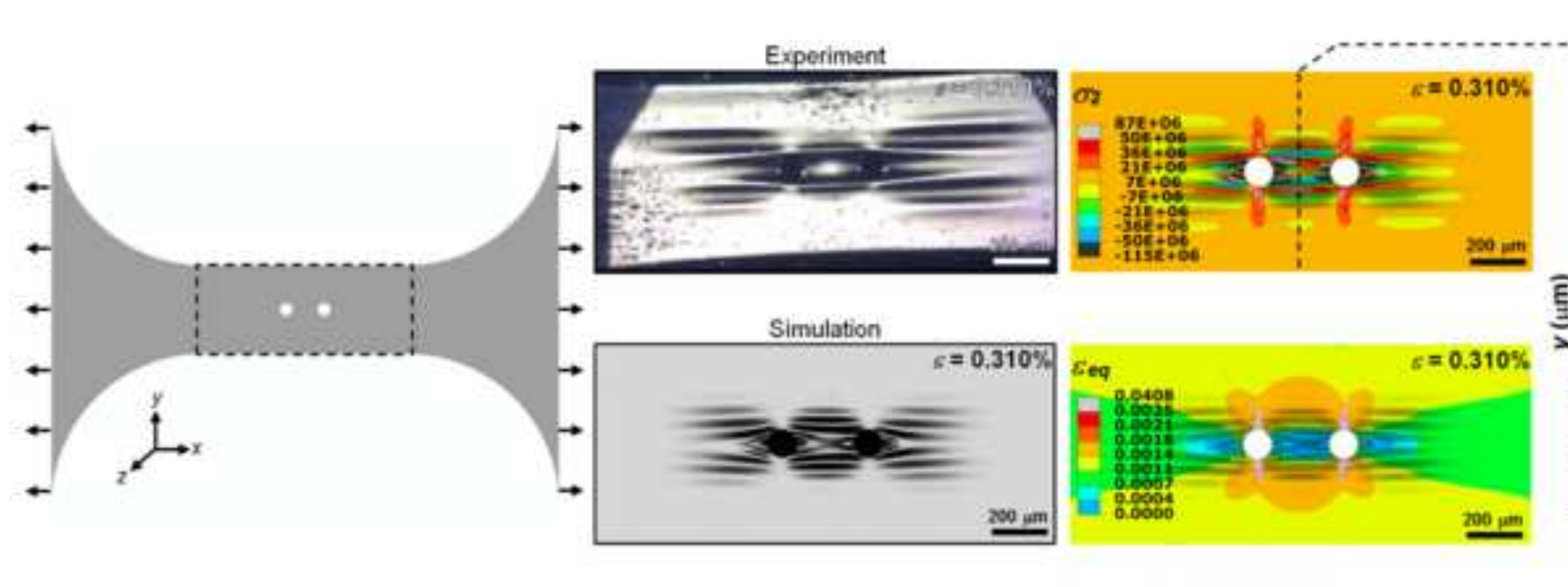

Out-of-plane displacoment

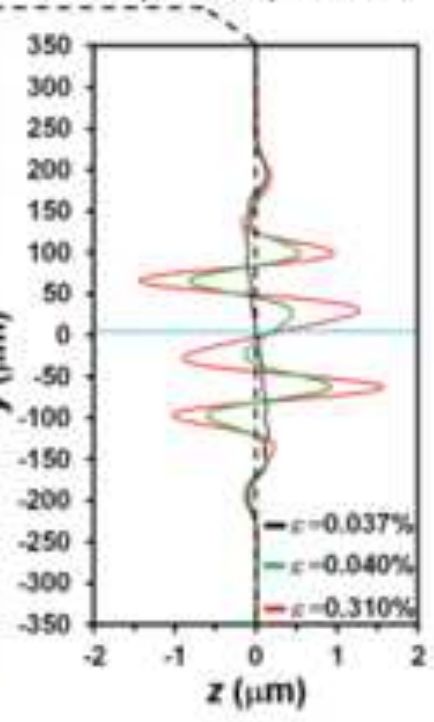

